\title{
Two-photon absorption: transitions induced in highly symmetric molecules by a magnetic field
}

\author{
David L Andrews \\ School of Chemical Sciences, University of East Anglia, Norwich NR4 7TJ, England \\ Received 6 May 1986
}

\begin{abstract}
It has recently been shown that certain two-photon transitions can be induced in atoms by applying a static magnetic field at the intersection of two crossed laser beams. The mechanism involves quantum mechanical interference between different excitation pathways. In this letter it is shown that a similar effect takes place in any highly symmetric molecule such as methane or sulphur hexafluoride belonging to a cubic symmetry group. However, in this case the effect occurs through a non-linear magneto-optical interaction which is quite distinct from the interference mechanism.
\end{abstract}

A recent paper by Diebold (1985) has considered the case of two-beam two-photon absorption in atoms, in which transitions provide access to excited states corresponding to the sum of the absorbed photon energies. Attention was drawn to the fact that two-photon ${ }^{1} \mathrm{~S}-{ }^{1} \mathrm{~S}$ transitions, which are normally forbidden if the electric vectors of the two beams are perpendicular, can nonetheless become allowed if a static magnetic field is applied. The mechanism for this phenomenon involves quantum mechanical interference between different pathways through ${ }^{1} \mathrm{P}$ intermediate excited states, by virtue of the removal of spin degeneracy by the magnetic field.

It is the purpose of this letter to show that a similar effect takes place in highly symmetric molecules such as methane or sulphur hexafluoride which belong to one of the cubic or icosahedral symmetry point groups. Here too, two-photon transitions to excited states belonging to the totally symmetric representation are forbidden when the two beams have perpendicular electric vectors, but are allowed when a mutually perpendicular magnetic field is applied. In this case, however, the effect occurs through a non-linear magneto-optical interaction. This process can take place irrespective of any lifting of spin degeneracy by the magnetic field, and quantum mechanical interference is not implicated in the mechanism.

The matrix element $M_{f 0}$ for a two-photon transition $|f\rangle \leftarrow|0\rangle$ in the presence of a static magnetic field $\boldsymbol{B}$ can be obtained from second-order time-dependent perturbation theory, using dressed basis states given by

$$
\left|r^{\prime}\right\rangle=|r\rangle-\sum_{s \neq r}\left(\boldsymbol{m}^{s r} \cdot \boldsymbol{B}\right) E_{r s}^{-1}|s\rangle
$$

where $E_{r s}$ is the difference in the zeroth-order energies $E_{r}-E_{s}$, and $\boldsymbol{m}^{s r}$ is the magnetic dipole transition moment for the transition $|s\rangle \leftarrow|r\rangle$. The leading term in the result for the matrix element can be expressed using the implied summation convention for repeated indices as

$$
M_{f 0}=\left(1 / 2 c \varepsilon_{0}\right)^{-1}\left(I_{1} I_{2}\right)^{1 / 2}\left(S_{i j} e_{1 i} e_{2 j}+T_{i j k} e_{1 i} e_{2 j} B_{k}+\ldots\right) .
$$


Here $e_{1}, e_{2}$ denote the orthogonal plane polarisation vectors of the two beams with intensities $I_{1}, I_{2}$, and frequencies $\omega_{1}, \omega_{2}$ respectively, where

$$
E_{f 0}=\hbar \omega_{1}+\hbar \omega_{2} \quad\left(\omega_{1} \neq \omega_{2}\right)
$$

$S_{i j}$ is the usual molecular two-photon absorption tensor and $T_{i j k}$ is a third-rank molecular tensor. These tensors are defined as follows:

$$
\begin{aligned}
S_{i j}=\sum_{r}\left(\frac{\mu_{i}^{f r} \mu_{j}^{r 0}}{E_{r 0}-\hbar \omega_{2}}+\frac{\mu_{j}^{f r} \mu_{i}^{r 0}}{E_{r 0}-\hbar \omega_{1}}\right) \\
T_{i j k}=\sum_{r, s}\left(\frac{\mu_{i}^{f s} \mu_{j}^{s r} m_{k}^{r 0}}{\left(E_{s 0}-\hbar \omega_{2}\right) E_{r 0}}+\frac{\mu_{i}^{f s} m_{k}^{s r} \mu_{j}^{r 0}}{\left(E_{s 0}-\hbar \omega_{2}\right)\left(E_{r 0}-\hbar \omega_{2}\right)}\right. \\
+\frac{m_{k}^{f s} \mu_{i}^{s r} \mu_{j}^{r 0}}{\left(E_{s 0}-\hbar \omega_{1}-\hbar \omega_{2}\right)\left(E_{r 0}-\hbar \omega_{2}\right)}+\frac{\mu_{j}^{f s} \mu_{i}^{s r} m_{k}^{r 0}}{\left(E_{s 0}-\hbar \omega_{1}\right) E_{r 0}} \\
\left.+\frac{\mu_{j}^{f s} m_{k}^{s r} \mu_{i}^{r 0}}{\left(E_{s 0}-\hbar \omega_{1}\right)\left(E_{r 0}-\hbar \omega_{1}\right)}+\frac{m_{k}^{f s} \mu_{j}^{s r} \mu_{i}^{r 0}}{\left(E_{s 0}-\hbar \omega_{1}-\hbar \omega_{2}\right)\left(E_{r 0}-\hbar \omega_{1}\right)}\right)
\end{aligned}
$$

where $\mu^{s r}$ denotes an electric dipole transition moment. Whilst other terms in equation (5) result from dressing by the magnetic field of the initial and final states for the two-photon transition, the second and fifth terms represent the dressing effect on a virtual intermediate state $|r\rangle$. In the special case where there is a one-photon nearresonance with a spin state whose degeneracy is lifted by the field, these two terms are associated with the effect discussed by Diebold. For our purposes here, however, there is no specific need for the involvement of either near-resonance or degeneracy lifting. For later reference, we note that the transformation properties of the tensors defined by equations (4) and (5) under the operations of the full rotation group are given by

$$
\begin{aligned}
& D^{(1-)} \otimes D^{(1-)}=D^{(0+)} \oplus D^{(1+)} \oplus D^{(2+)} \\
& D^{(1-)} \otimes D^{(1-)} \otimes D^{(1-)}=D^{(0+)} \oplus 3 D^{(1+)} \oplus 2 D^{(2+)} \oplus D^{(3+)}
\end{aligned}
$$

for $S_{i j}$ and $T_{i j k}$ respectively.

The rate $\Gamma$ of two-photon absorption can be calculated from equation (2) using the Fermi golden rule:

$$
\Gamma=(2 \pi / \hbar)\left|M_{f i}\right|^{2} \rho_{f}
$$

where $\rho_{f}$ is the density of final states. The leading term is independent of the magnetic field and comes from $\left|S_{i j} e_{1 i} e_{2 j}\right|^{2}$. After effecting a rotational average to account for the random molecular orientations in a fluid phase (Andrews and Thirunamachandran 1977), this term leads to the result

$$
\Gamma=\frac{I_{1} I_{2} \pi \rho_{f}}{60 \hbar c^{2} \varepsilon_{0}^{2}}\left(4 S_{\lambda \mu} \bar{S}_{\lambda \mu}-S_{\lambda \lambda} \bar{S}_{\mu \mu}-S_{\lambda \mu} \bar{S}_{\mu \lambda}\right)
$$

for the special case where $e_{1}$ is perpendicular to $e_{2}$. Rewriting the result explicitly in terms of irreducible Cartesian tensors (Jerphagnon et al 1978), we obtain

$$
\Gamma=\frac{I_{1} I_{2} \pi \rho_{f}}{60 \hbar c^{2} \varepsilon_{0}^{2}}\left(5 S_{\lambda \mu}^{(1+)} \bar{S}_{\lambda \mu}^{(1+)}+3 S_{\lambda \mu}^{(2+)} \bar{S}_{\lambda \mu}^{(2+)}\right)
$$


Here $S_{\lambda \mu}^{(1+)}$ is the weight-1 even-parity axial part of the tensor $S_{\lambda \mu}$, whose components have the transformation properties of rotations, and $S_{\lambda \mu}^{(2+)}$ is the traceless weight-2 part of $S_{\lambda \mu}$. It is evident from the result that there is no term involving $S_{\lambda \mu}^{(0+)}$, which alone transforms as a scalar, although such a term does normally arise in other polarisation configurations. For any two-photon transition to a state belonging to the totally symmetric representation in a molecule belonging to one of the cubic or icosahedral point groups $T, T_{h}, T_{d}, O, O_{h}, I$ or $I_{h}$, the only tensor components which are not identically zero are those which transform as a scalar; hence under the conditions described, the two-photon absorption is forbidden in the absence of a magnetic field. This conclusion has exactly the same physical basis as the more familiar result that the Raman bands associated with totally symmetric vibrations in cubic or icosahedral molecules are fully polarised (Koningstein 1972).

In the presence of a magnetic field, the leading correction to equation (10) from the expansion of equation (8) comes from the two cross terms involving $S_{i j} e_{1 i} e_{2 j} \bar{T}_{l m n} e_{11} e_{2 m} B_{n}$ and its complex conjugate. However, if we specify the direction of the magnetic field by

$$
\hat{\boldsymbol{B}}=\boldsymbol{e}_{1} \times \boldsymbol{e}_{2}
$$

so that the three vectors $e_{1}, e_{2}$ and $B$ are mutually orthogonal, then the rotational average of these two cross terms gives a vanishing result. However, the succeeding correction term resulting from $\left|T_{i j k} e_{1 i} e_{2 j} B_{k}\right|^{2}$ can be evaluated and after rotational averaging gives

$$
\begin{aligned}
\Gamma^{\prime}=\frac{I_{1} I_{2}|\boldsymbol{B}|^{2} \pi \rho_{f}}{420 \hbar c^{2} \varepsilon_{0}^{2}} & \left(2 T_{\lambda \lambda \mu} \bar{T}_{\mu \nu \nu}+2 T_{\lambda \lambda \mu} \bar{T}_{\nu \mu \nu}-5 T_{\lambda \lambda \mu} \bar{T}_{\nu \nu \mu}+2 T_{\lambda \mu \lambda} \bar{T}_{\mu \nu \nu}\right. \\
& -5 T_{\lambda \mu \lambda} \bar{T}_{\nu \mu \nu}+2 T_{\lambda \mu \lambda} \bar{T}_{\nu \nu \mu}-5 T_{\lambda \mu \mu} \bar{T}_{\lambda \nu \nu}+16 T_{\lambda \mu \nu} \bar{T}_{\lambda \mu \nu} \\
& -5 T_{\lambda \mu \nu} \bar{T}_{\lambda \nu \mu}+2 T_{\lambda \mu \mu} \bar{T}_{\nu \lambda \nu}-5 T_{\lambda \mu \nu} \bar{T}_{\mu \lambda \nu}+2 T_{\lambda \mu \nu} \bar{T}_{\nu \lambda \mu} \\
& \left.+2 T_{\lambda \mu \mu} \bar{T}_{\nu \nu \lambda}+2 T_{\lambda \mu \nu} \bar{T}_{\mu \nu \lambda}-5 T_{\lambda \mu \nu} \bar{T}_{\nu \mu \lambda}\right) .
\end{aligned}
$$

This expression can be recast in terms of the appropriate third-rank irreducible tensors using equation (2.11) from Andrews and Wilkes (1985), and the result is as follows:

$$
\Gamma^{\prime}=\frac{I_{1} I_{2}|\boldsymbol{B}|^{2} \pi \rho_{f}}{420 \hbar c^{2} \varepsilon_{0}^{2}}\left(5 T_{\lambda \mu \nu}^{(0+)} \bar{T}_{\lambda \mu \nu}^{(0+)}+14 T_{\lambda \mu \nu}^{(2+)} \bar{T}_{\lambda \mu \nu}^{(2+)}+5 T_{\lambda \mu \nu}^{(3+)} \bar{T}_{\lambda \mu \nu}^{(3+)}\right) .
$$

The crucial feature of this result is the appearance of the weight- 0 tensor $T_{\lambda \mu \nu}^{(0+)}$, which persists even for transitions to totally symmetric states of tetrahedral, octahedral or icosahedral molecules.

The magnitude of the effect represented by this result can be estimated by noting that compared with the usual two-photon rate of equation (10), the various terms in the expression for the field-induced rate each contain an additional factor of the order of $\kappa^{2}=\left(\boldsymbol{m}^{p q} \cdot \boldsymbol{B} / \Delta E\right)^{2}$, where $\boldsymbol{m}^{p q}$ is a typical magnetic dipole transition moment and $\Delta E$ a typical detuning from resonance. Thus if $\left|\boldsymbol{m}^{p q}\right| \sim 1$ Bohr magneton, $|\boldsymbol{B}| \sim 1 \mathrm{~T}$ and $\Delta E \sim 5 \times 10^{13} \mathrm{~Hz}$, the value of $\kappa$ is approximately $3 \times 10^{-4}$, and so the rate is smaller by a factor of approximately $10^{7}$ than that usually associated with two-photon absorption. This represents a signal level which is still appreciably larger than that associated with three- and four-photon absorption spectroscopy, which is becoming an increasingly well established experimental technique (Levenson 1983). However, it is also worth noting that since the two-photon result depends on the intensity product $I_{1} I_{2}$, 
then increasing the intensity of each beam by a factor of the order of $\kappa$ can restore the field-induced absorption signal to its former level. An intensity increase of this order is readily attainable by the use of $Q$-switched lasers in conjunction with modelocking techniques. Hence the effect should certainly be measurable with current laser instrumentation.

In conclusion, it has been shown that in the particular laser beam geometry described, the application of a static magnetic field can induce two-photon molecular transitions that are forbidden in its absence. The mechanism, which is not specifically molecular but in principle also applies to atoms, is a non-linear magneto-optical interaction mediated by the response tensor defined in equation (5). In contrast to the process discussed by Diebold, this mechanism does not involve the removal of spin degeneracy in intermediate states.

\section{References}

Andrews D L and Thirunamachandran T 1977 J. Chem. Phys. 675026

Andrews D L and Wilkes P J 1985 J. Chem. Phys. 832009

Diebold G J 1985 Phys. Rev. A 322739

Jerphagnon J, Chemla D and Bonneville R 1978 Adv. Phys. 27609

Koningstein J A 1972 Introduction to the Theory of the Raman Effect (Dordrecht: Reidel) p 130

Levenson M D 1983 Introduction to Nonlinear Laser Spectroscopy (New York: Academic) ch 5 